\title{
Design of a Structured Light Sensor for In Situ Calibration ${ }^{\dagger}$
}

\author{
Fred W. DePiero
}

Computer Vision and Robotics

Research Laboratory

Electrical and Computer Engineering Dept. The University of Tennessee

Knoxville, TN 37996-2100
Reid L. Kress

Robotics

and Process Systems Division

Oak Ridge National Laboratory

P.O. Box 2008, Bldg. 7601

Oak Ridge, TN 37831-6304

\begin{abstract}
This paper highlights a ranging sensor that can be calibrated at standoffs of 1 to 3 feet with a depth of field of $\approx 1 \mathrm{ft}$. Tests at a 16 " standoff have produced a 0.004 inch standard deviation in height measurement errors. The data density and feld of view are programmable. The sensor is positioned robotically, allowing it to provide accurate, short-range measurements prior to manipulation tasks. The sensor head is relatively inexpensive $(\approx \$ 10 k)$ and is designed for in situ calibration. This approach is intended to allow replacement components to be installed, calibrated, and validated without decontamination. Calibration, validation and data acquisition are all designed to be performed remotely and in a highly automated fashion. These in situ features of the design are targeted for the interest of industry with the aim of producing a sensor having a longer lifetime and a lower maintenance cost.
\end{abstract}

\section{Introduction}

Oak Ridge National Laboratory and the University of Tennessee maintain ongoing developments in novel ranging sensors for use in the Department of Energy's Waste Processing Operations, as part of the environmental restoration and waste management (ERWM) program. The Surface Characterization and Object Pose Equipment (SCOPE) has been designed for range image acquisition in hazardous environments. Its primary purpose is to aide in the repackaging of waste materials in automated gloveboxes. It is also capable of performing surface inspections of the exterior of containment vessels that house hazardous materials.

This paper describes the method of range acquisition and the in situ calibration of the sensor. A Structured Light approach to ranging [1][2] was chosen for SCOPE because it provides a low cost and accurate sensor head which can be easily and reliably calibrated. Previous Structured Light sensors developed by ORNL [3] [4] have performed well, but did lack in situ refit of components.

\footnotetext{
${ }^{0}+$ Research sponsored by the Office of Technology Development, U.S. Department of Energy, and managed by Martin Marietta Energy Systems, Inc., under contract DE-AC0584 OR21400
}

An active laser-based method of ranging was needed for SCOPE because a significant percentage of items in the waste repackaging stream are contained in plastic bags. These objects include tools, small electrical parts such as motors and transducers, gloves and boots, see Fig. 1. An earlier stereo-based method of ranging had some difficulty with bagged objects. Laser range finders were not used for SCOPE because of their higher cost and inability to accommodate remote refit and recalibration. Their higher speed would have been a benefit in general, however it was not strictly required, as a 15 second acquisition time was targeted for SCOPE. Structured Light also has the advantage of being able to yield a system with a wide range of standoffs and accuracies. A target accuracy of 0.030 inches was set for the range data.

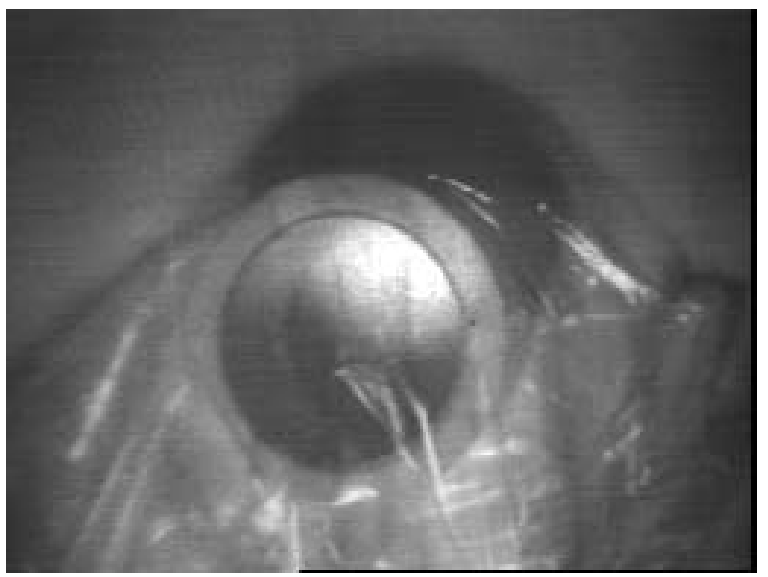

Figure 1: Typical bagged item in waste stream.

The basic sensor design is illustrated in Fig. 2. A line-generating laser produced a plane of light that emanated from a cylindrical lens attached to the body of the unit. The diode laser and lens formed a compact and rugged device and are readily available as a commercial unit.

It was desired to reposition the laser rapidly and reliably. For this purpose a rotating mirror was se- 
lected. This was a high precision device with a galvanometeric-based operation. It was commercially available and complete with a digital interface to its servo controller.

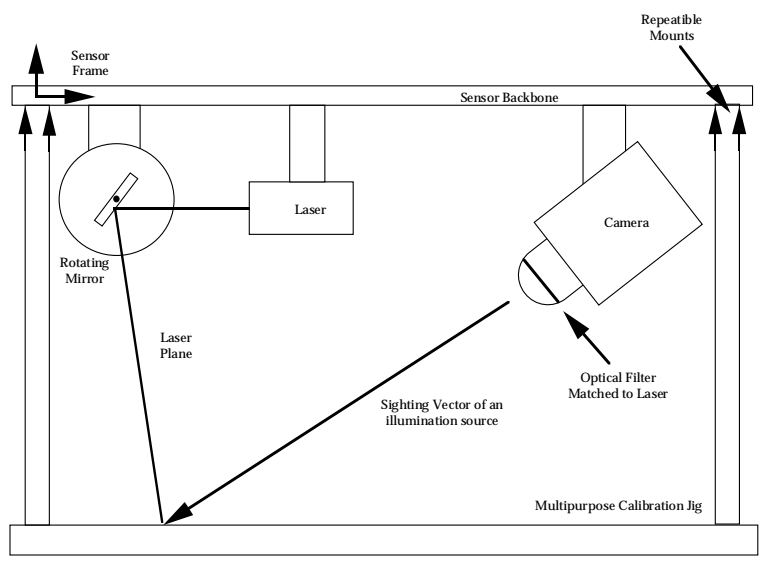

Figure 2: Basic sensor design

\section{Real-Time Range Data Acquisition}

A method for real-time range data acquisition was developed for SCOPE that helped reduce the problems associated with spurious reflections of the laser plane. A reflection of the laser plane off of unknown objects can cause significant errors in range data. The data acquisition process was referred to as "Gray-Level Position Stamping" (GLPS). This involved the accumulation of two images. The first was a gray scale image that looks like a time lapse version of what the camera sees. See Fig. 3. It contains closely spaced occurences of the laser as it was observed intersecting objects in the scene. Having a single image with many laser lines is very advantageous from a processing speed point of view simply because it is very rich with data. However, this image alone is useless without accompanying information describing the laser's position. These data are provided in the GLPS image. This is a processed version of the first which has been thresholded and then position-stamped with the laser's location. To generate the GLPS image each pixel is first compared to a threshold. If it exceeded the given limit, its image location is replaced with a position stamp value, else zero. A separate array was formed during a scan that associated the GLPS values with a description of the laser plane position. Since the GLPS image was simply a processed version of the first, the two are registered with each other at the pixel level.

The rotating mirror selected for SCOPE had a settling time of $0.8 \mathrm{mS}$ for small excursions. Mirror positioning and the camera's electronic shutter were orchestrated in a manner such that the mirror was only moved while the shutter was closed. This allowed range data to be accumulated at the camera's frame rate. The computing platform was VME-based. It consisted of a Motorola processor board with 68030 CPU and a DataCube MV20 image processing board. An optical filter was used on the camera that matched the laser's optical frequency. This, together with ample laser power produced images with favorable signal to noise ratios. The filters permitted normal flourescent room lighting to be used without interfering with the range acquisition.

To eliminate spurious reflections a minimum and a maximum version of the GLPS data was generated. Imagine the process of sweeping the laser across the cameras field of view in a continuous motion. Because of the finite thickness of the laser plane there will be some earliest and lastest laser position that a given pixel would be illuminated. If the laser were to take sufficiently large steps and no blurring occurs, then the earliest and latest position are identical. However if a spurious reflection of the laser plane occurs during the sweep then the nominal progression of positions in the image are disrupted.

The earliest and latest laser positions in which a pixel is illuminated are recorded in two separate images, referred to as the minimum and maximum GLPS, respectively. When the laser progresses normally across the camera's view there was only a small change between the minimum and maximum GLPS values at a given pixel. When a spurious reflection occurs the laser plane can be displaced and reoriented. In this case the maximum - minimum GLPS position difference can become greater than a set threshold and the corresponding range point can be eliminated. When computing range points the mean of the GLPS extrema was used to find the laser position.

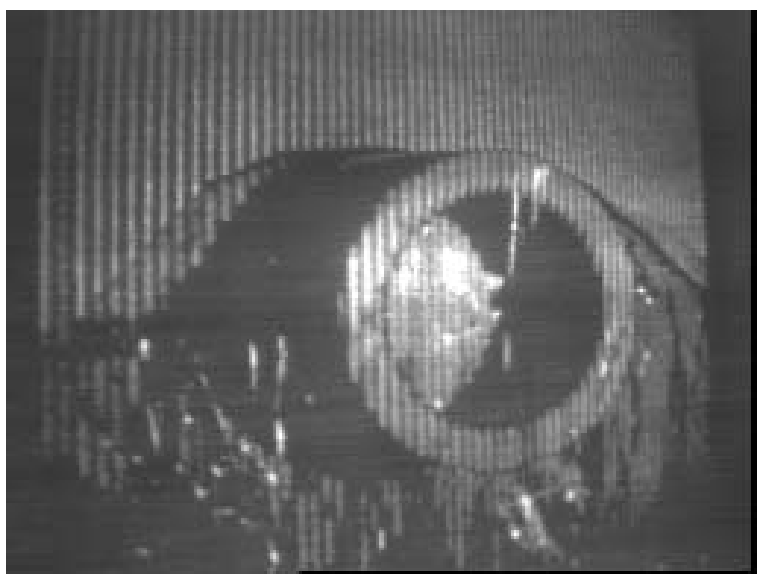

Figure 3: Accumulated gray scale image of scene illuminated by many laser lines.

\section{Range Point Calculation}

This process began by searching the gray scale image in a horizontal direction so that the images of the laser line are encountered in a near-orthogonal direction. See Fig. 3. The center of each laser line was computed via a mean weighted by the pixel intensity. The standard deviation was also computed to help eliminate blurry laser lines - these cases needed to be dropped because the true location of the laser 


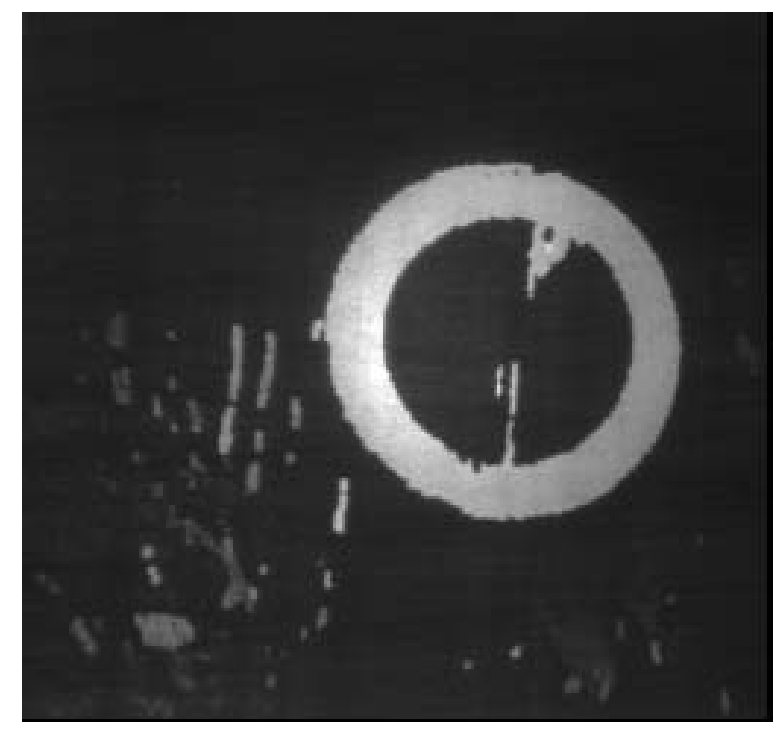

Figure 4: Range data acquired by SCOPE.

line could not be recovered reliably when the laser light was smeared in the image.

Having determined an image coordinate at the center of the laser line, a 'sighting vector' was found using the camera model. See Fig. 5. The camera model was used to find two quantities, a point $B$ and a direction $v$, both with respect to the sensor's coordinate frame. If $B$ was used to locate the tail of $v$ then the illumination source could be located in space by following along $v$ an unknown distance $t$. The kinematic model must be employed at this point to find $N$ which is normal to the laser plane and has a length equal to the shortest distance from the plane to the origin. The method for finding $N$ is discussed below. The unknown $t$ can be found using

$$
n^{T}\left(B+t_{v}\right)
$$

and the relationship

$$
n=N /|N|
$$

to yield

$$
t=\left(|N|-n^{T} B\right) / n^{T} v
$$

This calculation allows a single range point to be found. Many such points can be found using an image such as Fig. 3 together with its associated GLPS data. The range data density is selectable via the choice of the angular increment of the rotating mirror and by the number of rows examined in the gray scale image.

Note that the sensitivity of range calculations depends on the product $n^{T} v$. These vectors describe the normal to the laser plane and the direction of the sighting vector, respectively. When these become nearly orthogonal the sensitivity to noise in their components increases dramatically. By virtue of SCOPE's size having a separation between the mirror and camera of 12 inches and its scanning requirements, the straight line deviation between these vectors never exceeded more than 30 degrees. Hence SCOPE's performance was not significantly impacted by the numerical sensitivity of the ranging calculation.

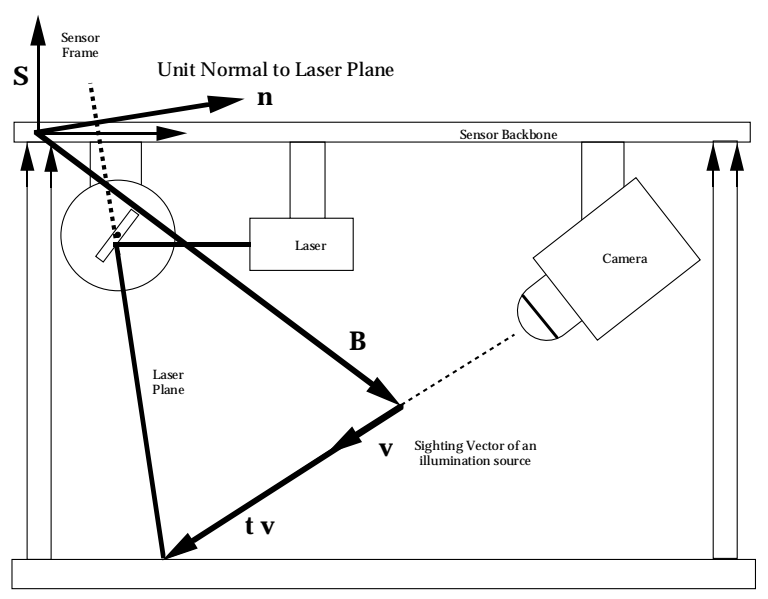

Figure 5: Range point calculation.

The kinematic model was needed to determine $N$, which locates the laser plane relative to the sensor frame $S$. Fig. 6 depicts a number of intermediate coordinate frames that were used in the model. The laser plane was described relative to frame $L$ using two vectors and a point. The point was coincident with the origin of frame $L$ and was at the center of the laser's cylindrical lens. The two vectors lie in the $\mathrm{x}-\mathrm{y}$ plane of $L$ with each of their tails at the origin of $L$. These two vectors are $d_{1}$ and $d_{2}$ in the figure and were referred to as the 'dark spot vectors' for reasons that will become more clear in the section on kinematic modeling.

It was then necessary to locate the surface of the rotating mirror and to determine the orientation of the reflected laser plane. The center of the rotating shaft that turns the mirror was described by frame $A$. The mirror's surface was found by rotating the frame $M$ to the current shaft orientation and then translating $M$ along its $-z$ direction until $M$ 's x-y plane was coincident with the mirror's reflecting surface. The intersection of $d_{1}$ and $d_{2}$ with the plane of the mirror was found via a method similar to that of finding the intersection of the sighting vector with the laser plane, as described above. The result of this intersection was an extension of $d_{1}, d_{2}$ out to the mirror to form $D_{1}$ and $D_{2}$, also in Fig. 6. The vector $D_{1}$ could then be decomposed into components that are parallel $\left(p_{1}\right)$ and orthogonal $\left(o_{1}\right)$ to the mirror.

$$
o_{1}=\left(M_{z}^{T} D_{1}\right) M_{z} p_{1}=D_{1}-o_{1}
$$

Where $M_{z}$ was the unit vector along the z-axis of frame $M$. This allowed points $R_{1}$ and $R_{2}$ in the reflected plane to be found with

$$
R_{1}=D_{1}+p_{1}-o_{1} .
$$

$R_{2}$ was found in a similar manner and then each were transformed to be expressed with respect to Frame $S$. 
If the $D_{i}$ vectors were also expressed with respect to $S$ then the laser plane was described by

$$
\begin{gathered}
N^{\prime}=\left(R_{1}-D_{1}\right) *\left(R_{2}-D_{2}\right) \\
n=N^{\prime} /\left|N^{\prime}\right| \\
|N|=n^{T} R_{1}
\end{gathered}
$$

where $*$ denotes the cross product.

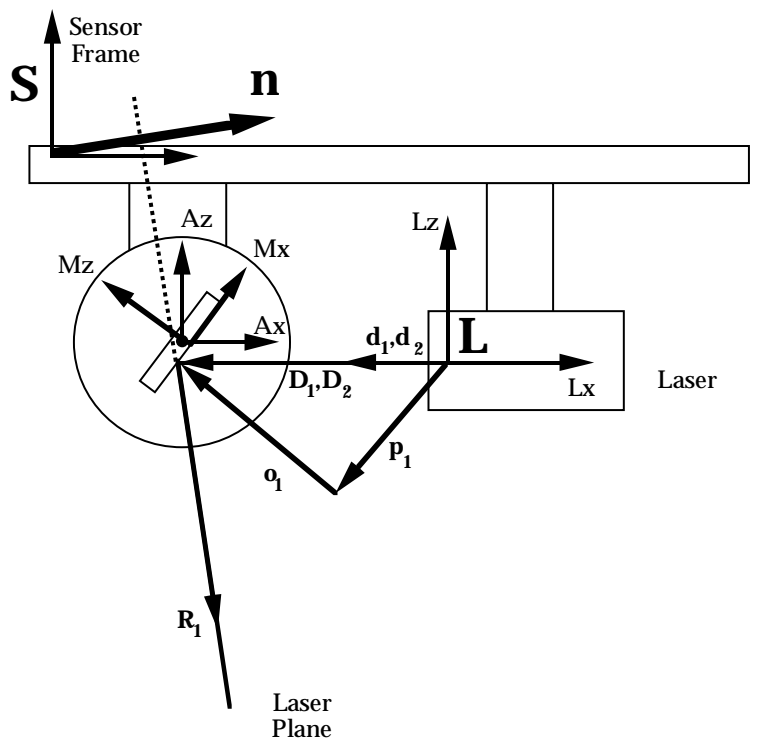

Figure 6: Kinematic parameters involved in range point calculations.

Despite the best efforts to produce accurate camera and kinematic models, measurement errors persisted when the two models were combined. This was observed when range points were collected across a machined plate. The measured surface of the plate appeared to be slightly bowl-shaped. Some of this remaining range error was reduced using a linear model, known as a systemic correction model. The height (z) coordinate of range measurements was corrected in this manner on SCOPE.

The correction was a function of the position of the range point

$$
\delta z=f(x, y, z)
$$

Several forms of models were examined. The computation of the model parameters and associated procedure are described below.

\section{Camera Calibration}

The purpose of camera calibration is to determine the precise direction of incoming illumination associated with each image pixel. The result of such a calibration is a model of the perspective effects and the distortions introduced by the camera. A description of the camera model, its use, and the calibration process is given in [5]. Note that subpixel accuracy was required in order to achieve the overall system performance. Calibration was performed using a multipurpose calibration jig. An online analysis and visualization tool was provided to help verify the suitability of camera models. The Two Planes method [6] was used with the guidance of [7] for selecting forms of the model.

\section{$5 \quad$ Kinematic Calibration}

The net result required from the kinematic model was a vector describing the location of the laser plane as a function of the motor position. This is denoted by $\mathrm{n}$ in Fig. 6. Computing this normal required precise values for a number of geometric parameters. A total of 15 parameters were needed for the model (Fig. 6).

The 6 degrees of freedom (DOF) associated with frames $L$ and $A$ are the typical sorts of parameters associated with a homogeneous coordinate transform, 3 translational and 3 rotational [8]. The mounting of the mirror on the motor shaft was assumed to have been accomplished with sufficient precision to require the modeling of only 2 DOF between frames $M$ and $A$. Introducing the dark spot vectors did increase the count of model parameters, however these vectors were useful in determining the direction of the laser plane after its reflection off the mirror. They also simplified the kinematic modeling procedure because parameter errors were manifested as 3-D Cartesian displacements, rather than as orientation errors in the laser plane.

Not all of the 15 parameters were optimized. A number of these were simply set to a convenient value (often zero) and any resultant kinematic effects were absorbed by other parameters. For example the $x$ displacement of the laser frame $L$ and $x$ displacement of the shaft frame $A$, did not both have to be optimized in order to achieve acceptable results. In all, 11 of 15 parameters were found via an iterative solution.

The Downhill Simplex algorithm [9] was used to optimize the kinematic parameters. It is one of many possible choices and was selected primarily because of its simplicity. The main advantage of this method was that it did not require a closed form for the partial derivatives of the cost function. This partial derivative would have been difficult to derive given the method used to reflect the laser off of the rotating mirror.

The cost function involved the difference between the observed and modeled position of the dark spot vectors. Actual dark spots were generated by suspending two small wires from the sensor housing. These wires intersected the laser plane prior to its reflection with the mirror. See Fig 7 . The centroid of the shadow produced by the each of the wires was described by the dark spot vectors discussed above. To collect the necessary input data for optimization, SCOPE was placed in a calibration jig at known standoffs above a series of machined plates. The plates were scanned with the protruding wires in place. Given the standoff and the current set of kinematic parameters, the location of each dark spot vector and its intersection with the calibration plate could be computed. The camera was employed to observe these darkened locations. The sighting vector of each dark spot was intersected with the plane of the calibration plate to find the observed 
Table 1: Degrees of Freedom (DOF) associated with kinematic model.

\begin{tabular}{|c||c|c|c|}
\hline Parameters & Reference Frame & DOF & Source of DOF \\
\hline \hline Frame L & S & 6 & Homogenous Coordinate Transform \\
\hline Dark Spot Vectors & $\mathrm{L}$ & 1 & Angle between vectors \\
\hline Frame A & $\mathrm{S}$ & 6 & Homogenous Coordinate Transform \\
\hline Frame M & $\mathrm{A}$ & 2 & Mirror thickness and Zero motor angle \\
\hline
\end{tabular}

location. The mean of the distances between each of the computed and observed dark spot centroids was used as the cost function. The simplex algorithm was typically presented with data on 40-50 distinct dark spots which were collected at 2 (or more) standoffs.

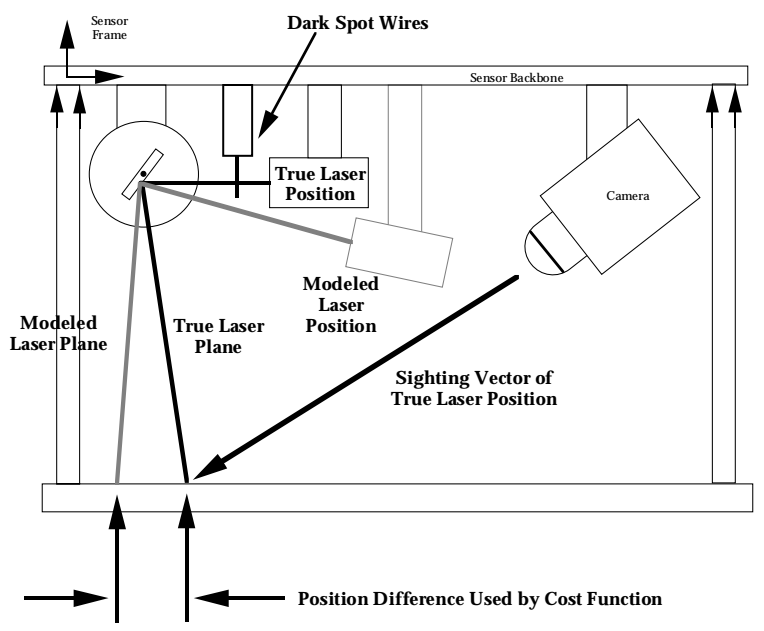

Figure 7: Iterative optimization of kinematic parameters.

Some low level image processing was necessary to find the dark spots in each camera image. The nominal appearance of the laser plane as it intersected each calibration plate was a line with slight curvature. A 3 rd order curve was fit to the center of this line. The curve was then traversed and an inverted gray scale weighting function was applied to find the centroid of each dark spot. Contributions to the centroid calculation were taken in the vicinity of the edges of each dark spot.

The wires responsible for creating the dark spots were removed after the kinematic calibration procedure to eliminate the missing regions of range data that would have otherwise resulted during sensing.

\section{$6 \quad$ Systemic Correction Model}

This model helped to reduce both the span of height errors and the standard deviation of these errors. The correction model was found be collecting measurements across a number of machined $z_{i}$ that were located at known standoffs. Once the height error $z_{i}$ and associated coordinate $(x, y, z)_{i}$ for $\mathrm{n}$ points were

$$
\delta z=C S
$$

here $z$ is $(n x 1)$ and formed with the $n$ height error examples. $C$ is formed with row vectors $C_{i}$ that contain the augmented world coordinates. Because of the resulting number of terms only low order models were considered. For example, a second order model had rows $C_{i}=$

$$
\left|\begin{array}{llllllllll}
1 & x & y & z & x^{2} & y^{2} & z^{2} & x y & x z & y z
\end{array}\right| \text {. }
$$

The correction model was found using SVD similar to the procedure for the camera models. Results of the systemic correction technique are given in Table 3 .

\section{Concluding Remarks}

SCOPE is a short-range measurement system applicable to several ERWM application areas. It has been used in two integrated demonstrations, one involving a prototype waste processing and separation plant in Savannah River Technology Center and the other involving an automated glovebox at Lawrence Livermore national Laboratory.

The techniques developed here have proven successful and can lead to the development of a sensor that could be refitted and recalibrated in situ without decontamination, resulting in a longer lifetime for the sensor and lower maintenance costs. The method of two planes has proven to be a robust and reliable method of camera calibration and very well suited to structured light sensors. The iterative method of kinematic calibration was successful, despite a fairly challenging number of degrees of freedom requiring estimation. The systemic correction technique was very simple to implement and yielded a respectable improvement in range accuracy. SCOPE performed reasonably well on tests involving bagged objects. Range errors can be seen in Fig. 4. These were primarily due to reflections within the bag which did not introduce a large enough GLPS difference to be filtered out.

SCOPE's performance at the time of the demonstration is summarized in Table 2. A first order systemic correction was used during these benchmarks, giving a 0.006 inch standard deviation to height errors. Table 3 summarizes the performance of the systemic correction for different order models.

\section{References}

[1] S. W. Holland, L. Rossol, and M. R. Ward. Computer Vision and Sensor-Based Robotics. Plenum Press, New York, 1978.

[2] B. K. P. Horn. Robot Vision. McGraw-Hill, Cambridge, MA, 1984. 
Table 2: Data acquisition performance of SCOPE.

\begin{tabular}{|c||c|c|}
\hline $\begin{array}{c}\text { Resolution } \\
\text { (pixels) }\end{array}$ & $\begin{array}{c}\text { Lateral Spacing } \\
\text { of Range Pts. (in.) }\end{array}$ & $\begin{array}{c}\text { Frame Capture Speed } \\
\text { (sec.) }\end{array}$ \\
\hline \hline $64 \times 64$ & 0.281 & 2.1 \\
\hline $128 \times 128$ & 0.141 & 4.3 \\
\hline $256 \times 256$ & 0.070 & 8.5 \\
\hline $512 \times 512$ & 0.035 & 17.1 \\
\hline
\end{tabular}

Table 3: Performance of the Systemic Correction Model

\begin{tabular}{|c||c|c|}
\hline Model Type & $\begin{array}{c}\text { Span of Height Errors } \\
(0.001 \text { in. })\end{array}$ & $\begin{array}{c}\text { Std. Dev. of Height Error } \\
(0.001 \text { in. })\end{array}$ \\
\hline \hline Nominal & 40 & 8 \\
\hline 1st Order & 30 & 6 \\
\hline 2nd Order & 22 & 4 \\
\hline
\end{tabular}

[3] B. L. Burks, F. W. DePiero, J. C. Rowe, C. B. Selleck, D. L. Jacoboski, and R. Markus. Generation of 3 surface maps in waste storage silos using a structured light source. In Proceedings of The Space Operations, Applications, and Research Symposium, Houston, 1991. NASA.

[4] B. L. Burks, F. W. DePiero, J. C. Rowe, C. B. Selleck, and D. L. Jacoboski. Final results of the application of a structured light source for surface mapping of the fernald k65 silos. In Proceedings of The American Nuclear Society, Boston, June 1992. ANS.

[5] F. W. DePiero and R. L. Kress. Camera calibration in a hazardous environment performed in situ with automated analysis and verification. In Proceedings of ANS Fifth Topical Meeting on Robotics and Remote Systems, Knoxville, TN, April 1993. ANS.

[6] A. Isaguirre, P. Pu, and J. Summers. A new development in camera calibration: Calibrating a pair of mobile cameras. Technical report, Department of Computer and Information Science, GRASP Laboratory, University of Pennsylvania, Philadelphia, 1985.

[7] G-Q. Wei and S. De Ma. Two plane camera calibration: A unified model. Technical report, National Laboratory of Pattern Recognition, Institute of Automation, Chinese Academy of Sciences, Bejing, 1991.

[8] H. Asada and J.-J. E. Slotine. Robot Analy sis and Control. John Wiley and Sons, New York, 1986.

[9] W. H. Press, B. P. Flannery, S. A. Teukolsky, and W. T. Vetterling. Numerical Recipes in C. Cambridge University Press, Cambridge, New York, 1988. 